\title{
Epigenetic alterations in hippocampus of SAMP8 senescent mice and modulation by voluntary physical exercise
}

\author{
Marta Cosín-Tomás ${ }^{1,2+}$, María J. Alvarez-López ${ }^{1,2+}$, Sandra Sanchez-Roige ${ }^{3}$, Jaume F. Lalanza ${ }^{3}$, \\ Sergi Bayod ${ }^{1}$, Coral Sanfeliu' ${ }^{2}$, Merce Pallàs ${ }^{1}$, Rosa M. Escorihuela ${ }^{3}$ and Perla Kaliman ${ }^{2 *}$ \\ 1 Unitat de Farmacologia, Facultat de Farmàcia Institut de Biomedicina Universitat de Barcelona (IBUB), Nucli Universitari de Pedralbes, Barcelona, Spain \\ ${ }^{2}$ Department of Brain Ischemia and Neurodegeneration, Institut d'Investigacions Biomèdiques de Barcelona (IIBB)-Consejo Superior de Investigaciones \\ Científicas (CSIC), Barcelona, Spain \\ ${ }^{3}$ Departamento de Psiquiatría y Medicina Legal, Facultad de Medicina, Instituto de Neurociencias, Universitat Autònoma de Barcelona, Barcelona, Spain
}

\section{Edited by:}

Rodrigo Orlando Kuljiš, Zdrav Mozak

Limitada, Chile

Reviewed by:

Ángel M. Carrión, Universidad Pablo de Olavide de Sevilla, Spain

Diego Ruano, University of Sevilla, Spain

*Correspondence:

Perla Kaliman, Department of Brain Ischemia and Neurodegeneration,

Institut d'Investigacions

Biomèdiques de Barcelona, Consejo

Superior de Investigaciones

Científicas, Rosselló 161, E-08036

Barcelona, Spain

e-mail:pkaliman@ub.edu

these authors have contributed

equally to this work.
The senescence-accelerated SAMP8 mouse model displays features of cognitive decline and Alzheimer's disease. With the purpose of identifying potential epigenetic markers involved in aging and neurodegeneration, here we analyzed the expression of 84 mature miRNAs, the expression of histone-acetylation regulatory genes and the global histone acetylation in the hippocampus of 8-month-old SAMP8 mice, using SAMR1 mice as control. We also examined the modulation of these parameters by 8 weeks of voluntary exercise. Twenty-one miRNAs were differentially expressed between sedentary SAMP8 and SAMR1 mice and seven miRNAs were responsive to exercise in both strains. SAMP8 mice showed alterations in genes involved in protein acetylation homeostasis such as Sirt 1 and Hdac6 and modulation of Hdac3 and Hdac5 gene expression by exercise. Global histone $\mathrm{H} 3$ acetylation levels were reduced in SAMP8 compared with SAMR1 mice and reached control levels in response to exercise. In sum, data presented here provide new candidate epigenetic markers for aging and neurodegeneration and suggest that exercise training may prevent or delay some epigenetic alterations associated with accelerated aging.

Keywords: exercise, aging, Alzheimer, SAMP8, microRNAs

\section{INTRODUCTION}

Epigenetic changes are currently recognized as part of the aging process and have been implicated in many age-related chronic diseases (Jakovcevski and Akbarian, 2012; Akbarian et al., 2013; Lopez-Otin et al., 2013). The term epigenetics includes a variety of processes known to regulate gene expression in a stable and potentially reversible way, without altering the primary DNA sequence (Jaenisch and Bird, 2003). The molecular mechanisms that mediate epigenetic regulation are principally DNA methylation, post-translational modifications of the histones, and regulation by non-coding RNAs. Among the latter, microRNAs (miRNAs) are small molecules (22 nucleotides approximately) that regulate gene expression by binding to its target messenger RNA (mRNA) inhibiting its translation, or, less frequently, promoting its degradation (Bartel, 2009). To date, around 700-800 miRNAs have been identified in the human genome (Bentwich et al., 2005). Altered expression of miRNAs has been described in different chronic pathologies and they are currently considered to be critical in the aging process (Jung and Suh, 2012). miRNAs seem to play an important role in the developing nervous system, in the physiology of high-order brain functions such as learning, memory, and emotion regulation, and in the manifestation of neurological disorders such as amyotrophic lateral sclerosis, Tourette's syndrome, Alzheimer's disease (AD) and others (Yang et al., 2007; Mastroeni et al., 2011; Goldie and Cairns, 2012; Van Den Hove et al., 2014). On the other hand, histone covalent modifications have also been implicated in the aging process (Dang et al., 2009; Greer et al., 2010; Siebold et al., 2010; Di Bernardo et al., 2012; Huidobro et al., 2013; Tammen et al., 2013). Histone acetyltransferases (HATs) and histone deacetylases (HDACs) are among the best characterized histone modifying enzymes in neurons (Crepaldi and Riccio, 2009). HATs transfer an acetyl group to the amino groups of histone lysine residues and generally increase DNA transcription. For their part, HDACs decrease DNA accessibility by deacetylation of histone lysines (Legube and Trouche, 2003). An adequate balance between HAT and HDAC levels and activity is crucial for neuronal homeostasis and for brain functions such as learning and memory (Saha and Pahan, 2006). Notably, alterations in histone acetylation levels have been observed in several models of neurodegenerative diseases, including AD (Scheff et al., 2007; Arendt, 2009).

It has been widely reported that the regular practice of physical exercise improves brain health and provides cognitive and psychological benefits (Kaliman et al., 2011). Some of the neurophysiological effects of physical exercise have been attributed to changes in the transcriptional profiles of growth and neurotrophic factors such as IGF1 and BDNF (Dishman et al., 2006; Gomez-Pinilla et al., 2008; Trejo et al., 2008; AlvarezLopez et al., 2013). Recent data have described the positive impact of physical exercise on epigenetic alterations in the rodent brain (Chandramohan et al., 2008; Collins et al., 2009; Abel and Rissman, 2013; Lovatel et al., 2013). 
The spontaneous senescence-accelerated P8 mouse model (SAMP8) is currently considered a model of AD (Pallas et al., 2008a; Morley et al., 2012b; Cheng et al., 2013a,b; Wang et al., 2013). Indeed, SAMP8 mice display cognitive and behavioral alterations which are accompanied by molecular features typical of $\mathrm{AD}$ such as overproduction of amyloid-beta protein, increased tau phosphorylation, cholinergic deficits in the forebrain and increased oxidative stress (Takeda, 2009; Del Valle et al., 2011; Morley et al., 2012a). SAMP8 mice were phenotypically selected from AKR/J, and SAM resistant mice (SAMR1), which have a similar genetic background, have been extensively used as a control model because they show normal aging characteristics (Takeda et al., 1991). With the purpose of identifying potential epigenetic markers involved in aging and neurodegeneration, here we studied the expression levels of a set of 84 mature miRNAs with reported effects on neurological development and disease, the expression of several genes involved in maintenance of the histone acetylation balance (HATs and HDACs) and the levels of histone global acetylation (H3ac, $\mathrm{H} 4 \mathrm{ac}$ ) in the hippocampus of 8-monthold SAMP8 and SAMR1 mice. We also explored the impact of 8 weeks of voluntary wheel running intervention on these parameters. Through these analyses, we identified hippocampal epigenetic factors that are altered in the senescent SAMP8 mice, some of which were modulated by physical exercise.

\section{MATERIALS AND METHODS}

\section{ANIMAL CARE AND VOLUNTARY EXERCISE PARADIGM}

All experimental procedures were approved by the Ethics Committee of the University of Barcelona (Comissió Ėtica d'Experimentació Animal UB), following the "Principles of laboratory animal care" and were carried out in accordance to the European Communities Council Directive (86/609/EEC).

SAMP8 and SAMR1 female mice were provided by El Parc Tecnològic (Barcelona, Spain) and were maintained under standard conditions (temperature $23 \pm 1^{\circ} \mathrm{C}$, humidity $50-60 \%$, 12:12-h light-dark cycle, lights on at 7:00 a.m.), with food (A04, Harlan, Spain) and tap water available ad libitum throughout the study. Body weight (g) was measured weekly. This study was performed in female mice as sex differences in the patterns of voluntary exercise in mice have been reported (Alvarez-Lopez et al., 2013).

The running wheels (ENV-044 Mouse Low-Profile Wireless Running Wheel, Med Associates Inc.; $15.5 \mathrm{~cm}$ circumference; $25^{\circ}$ from horizontal plane) were located in the animal colony room inside cages $19 \mathrm{~cm}$ high $\times 27 \mathrm{~cm}$ wide $\times 40 \mathrm{~cm}$ deep. Wheelrunning activity was monitored through a wireless transmitter system by using a Hub located in the same animal colony room. Wireless Running Wheel Manager Data Acquisition Software (SOF-860; Med Associates Inc.) recorded the activity and time of each wheel revolution, which occurred whenever a magnet attached to the wheel's axis made contact with an electronic switch sending a signal to the Hub. Although revolutions were monitored continuously, voluntary activity occurred primarily during the dark phase. The running mice were placed individually in the large cages and had unlimited access to a running wheel 7 days a week for 8 weeks. Control mice were placed individually in cages of equal size without a running wheel.
At the end of the intervention all mice were 8-month-old. Animals were sacrificed by decapitation and the brains were dissected on ice to obtain the hippocampus. Tissues were immediately frozen and stored at $-80^{\circ} \mathrm{C}$ for further analysis.

\section{PLASMA ANALYSIS}

Blood samples were collected in 5\% EDTA-tubes at the time of death for IGF1, cholesterol, and triglycerides determination. Plasma was obtained by centrifugation $(3500 \mathrm{rpm}, 10 \mathrm{~min}$, room temperature) and stored at $-80^{\circ} \mathrm{C}$.

IGF1 was determined by the ELISA kit Mouse/Rat Insulinlike Growth Factor-I; (Mediagnost, IGF-I EIA E25, Reutlingen, Germany) following the manufacturer's recommendations.

Plasma triglyceride and cholesterol concentrations were measured by using the colorimetric tests (Triglyceride L-type and Cholesterol kit, respectively), from Wako Chemicals $\mathrm{GmbH}$ (Neuss, Germany).

\section{TOTAL RNA EXTRACTION}

Total RNA was extracted using mirVana ${ }^{\mathrm{TM}}$ RNA Isolation Kit (Applied Biosystems) according to the instructions of the manufacturer. The yield, purity and quality of RNA were determined spectrophotometrically (NanoDrop, USA) and using the Bioanalyzer 2100 capillary electrophoresis. RNAs with 260/280 ratios and RIN higher than 1.9 and 7.5, respectively, were selected.

\section{REAL-TIME OUANTITATIVE PCR}

Random-primed cDNA synthesis was performed at $37^{\circ} \mathrm{C}$ starting with $0.3 \mu \mathrm{g}$ of RNA, using the High Capacity cDNA Archive kit (Applied Biosystems). Gene expression was measured in an ABI Prism 7900HT Real Time PCR system using TaqMan FAMlabeled specific probes (Applied Biosystems). A list of the probes used is presented in Supplementary Table 1 (S1). Results were normalized to TATA-binding protein (Tbp) expression.

\section{WESTERN BLOTS}

Histone fractions $(5 \mu \mathrm{g})$ were electrophoretically analyzed on $12 \%$ bis-Tris polyacrylamide gels and transferred to a $0.45 \mu \mathrm{m}$ PVDF membrane. Membranes were blocked for $1 \mathrm{~h}$ with 5\% BSA in PBS and incubated overnight at $4^{\circ} \mathrm{C}$ with the specific primary antibodies (1:1000, Millipore). Membranes were washed and incubated with peroxidase-labeled secondary antibodies at room temperature for $1 \mathrm{~h}$. Immunoreactive bands were detected by autoradiography. Specific bands from Western blot were quantified by scanning densitometry using Quantity One ${ }^{\circledR} 1-\mathrm{D}$ analysis 4.6.3. software (Bio-Rad USA, Life Science Research, Hercules, CA). Histone modifications levels were corrected by total histone expression.

\section{microRNA EXPRESSION ARRAY}

RNA samples from 16 female individuals (four from each group: sedentary SAMR1, runner SAMR1, sedentary SAMP8, runner SAMP8) were converted to CDNA through a reverse transcription reaction using miScript II RT Kit (Qiagen, Hilden Germany) according to the manufacturer's instructions. The expression of 84 mature miRNAs was then analyzed using the miScript ${ }^{\circledR}$ miRNA PCR Array-Neurological Development and Disease miRNA PCR Array (Qiagen). miRNAs expression was measured in an ABI 
Prism 7900HT through SYBR-green-based real time PCR. The data obtained were processed with the online software "Webbased miScript miRNA PCR Array data analysis tool." The mean of the relative gene expression of the small non-coding RNAs SNORD61, SNORD68, SNORD72, SNORD95, SNORD96A was used to normalize results since they presented similar expression levels across the individuals and groups and the lowest standard deviations among all the housekeeping miRNAs proposed.

\section{STATISTICAL ANALYSIS}

The statistical analysis was performed using the Statistical Package for Social Sciences (SPSS, version 19.0). The Two-Way ANOVA analysis of variance $[2$ strains $(\mathrm{R} 1, \mathrm{P} 8) \times 2$ conditions (sedentary, runner)] was conducted to assess strain and exercise intervention effects. Comparisons between groups were performed by two-tailed Student's $t$-test for independent samples; $p$-values below 0.05 were considered statistically significant. Statistical outliers ( $\geq$ two standard deviations from the mean) were removed from the analyses. Functional prediction analysis (Supplementary information S3-S6) was only performed for those miRNAs significantly altered with a $p<0.05$ and a magnitude of effect $\geq 1.4$.

\section{RESULTS}

\section{POSITIVE EFFECTS OF 8 WEEKS OF VOLUNTARY WHEEL RUNNING IN HIPPOCAMPAL GENE EXPRESSION AND IGF1 PLASMA LEVELS}

We analyzed the effects of 8 weeks of voluntary wheel running in 6-month-old SAMP8 and SAMR1 mice. Both strains displayed a similar and stable number of wheel revolutions/week over time throughout the intervention (SAMR1, $17762 \pm 2221.9$ average wheel revolutions/week; SAMP8, $18018 \pm 3035.7$ average wheel revolutions/week, Figure 1A). No changes were found in body weight, plasma triglycerides, or plasma cholesterol between the experimental groups (Figures 1B-D; Table 1).

To confirm the efficacy of the exercise intervention, we analyzed several factors previously reported to be modulated in rodents in response to physical training (e.g., plasma IGF1 and hippocampal Bdnf gene expression) (Kaliman et al., 2011). Both exercised SAMR1 and SAMP8 mice showed significantly increased IGF1 plasma levels compared with their corresponding sedentary groups (Figure 1E; Table 1).

In the hippocampus, $B d n f$ gene was underexpressed in sedentary SAMP8 compared with SAMR1 mice and both Bdnf and its receptor $\operatorname{TrkB}$ were significantly upregulated in response to the exercise intervention in both strains (Figures 1F,G; Table 1). Notably, after the exercise intervention $B d n f$ levels in SAMP8 mice were undistinguishable from those found in sedentary SAMR1 controls $\left[t_{(12)}=0.279, p=0.785\right]$ (Figure 1F). Neuritin gene, a well characterized target of BDNF, was upregulated in both strains by exercise training (Figure 1H; Table 1).

These results confirmed that the wheel running intervention was effective and therefore represents a good model to explore epigenetic effects of exercise in the SAMP8 mice.

\section{microRNA EXPRESSION PROFILE IS ALTERED IN SAMP8 HIPPOCAMPUS AND MODULATED BY PHYSICAL EXERCISE}

We compared the microRNA expression profiles between 8month-old SAMP8 and SAMR1 mice in order to identify putative senescence markers in the hippocampus. We used a miRNA PCR array which analyzes 84 different mouse miRNAs known to be altered in neurological diseases or involved in neuronal development. We found 18 miRNAs altered in sedentary SAMP8 compared with SAMR1 mice which were unresponsive to exercise, three miRNAs altered in SAMP8 and modulated by exercise and four miRNAs that were similarly expressed in SAMP8 and SAMR1 mice and modulated by exercise in both strains. TwoWay ANOVA analysis of this set of miRNAs are shown in Table 2. Statistical analysis of miRNAs similarly expressed in SAMP8 and SAMR1 mice and unresponsive to the exercise intervention are shown in Supplementary Table 2 (S2).

Among the miRNAs that were significantly upregulated in SAMP8 compared with SAMR1 mice, miR-30e-5p, miR-125b-5p, and miR-128-3p have also been reported to be upregulated in post-mortem human AD hippocampus (Lukiw, 2007; Cogswell et al., 2008). Similarly, we found an increased expression of let7i-5p, miR-29a-3p, miR-29c-3p, miR-30a-5p, miR-98-5p, miR138-5p, miR-139-5p, miR-140-5p, miR-146b-5p, miR-148b-3p, miR-181a-1-3p, miR-181a-5p, miR-194-5p, and miR-342-3p, all of which have been reported to be altered in different AD tissues (Cogswell et al., 2008; Hebert et al., 2008; Maes et al., 2009; Wang et al., 2011, 2012; Lau et al., 2013). The rest of the differentially expressed miRNAs between strains have been found to be altered in different neurodegenerative models (28a-5p, miR-337-3p, miR-431-5p, miR-455-5p). The functional information available in the literature for these miRNAs in the central nervous system (CNS) is summarized in Supplementary Table 3 (S3).

Interestingly, miR28a-5p, miR-98-5p, and miR-148b-3p expression was significantly higher in sedentary SAMP8 compared with sedentary SAMR1 mice and this difference was further accentuated by exercise (Figures 2A-C). In addition, we found that miR-7a-5p, miR-15b-5p, miR-105, and miR-133-3p exhibited similar expression levels in sedentary strains but were similarly modulated by exercise in SAMP8 and SAMR1 mice (Figures 2D-G). Functional information available in the literature for the role in the CNS of the miRNAs regulated by exercise is summarized in Supplementary Table 4 (S4).

\section{ALTERATIONS IN THE EXPRESSION OF HAT AND HDAC GENES AND IN GLOBAL HISTONE MODIFICATIONS IN HIPPOCAMPUS OF SEDENTARY SAMP8: EFFECTS OF EXERCISE}

Alterations in histone acetylation levels have been observed in several models of neurodegenerative diseases. Therefore, we analyzed in sedentary and exercised SAMP8 and SAMR1 mice, the hippocampal expression of the HAT P300 and the NAD+ dependent HDAC Sirt1, both of which have been implicated in AD pathogenesis (Pallas et al., 2008b; Min et al., 2010) as well as a group of NAD+ independent HDACs (Hdac1, Hdac2, Hdac3, Hdac5, Hdac6).

We did not find any differences between strains or any modulation with exercise in histone acetyltransferase $P 300$ gene expression (Table 3, Figure 3A). We found lower expression levels of the histone deacetylases Sirt1, Hdac5, and Hdac6 in sedentary SAMP8 compared with SAMR1 mice (Table 3, Figures 3B,F,G) while no significant differences between strains were detected for Hdacl, Hdac2 and Hdac3 (Table 3, Figures 3C-E). 


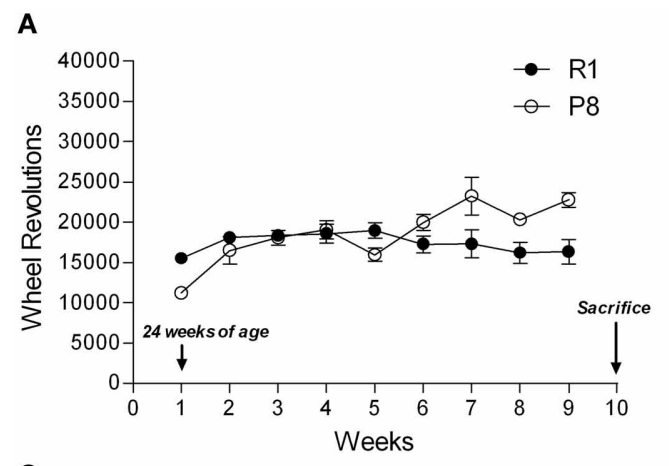

C

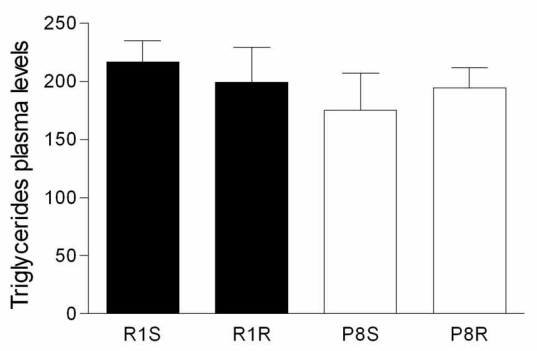

E

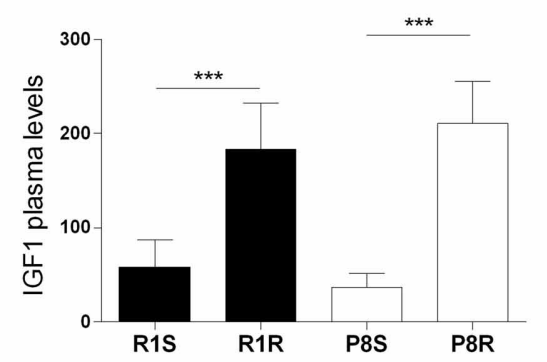

G

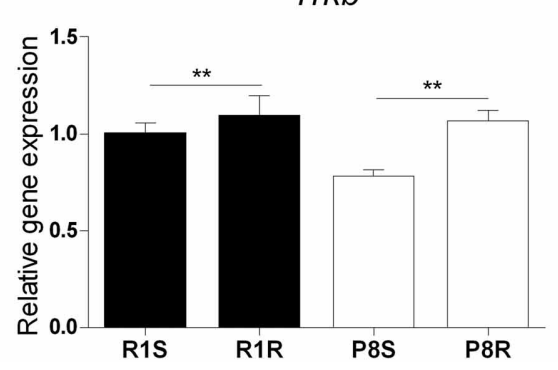

FIGURE 1 | Beneficial effects of 8 weeks of exercise training in SAMP8 senescent mice. (A) Mean wheel revolutions/week register in exercised SAMR1 and SAMP8 mice; (B) Body weight (g) mean during exercise intervention in sedentary and exercised groups from both SAMR1 and SAMP8 strains. (C) Triglyceride plasma levels (mg/dL). (D) Cholesterol plasma levels (mg/dL). (E) IGF1 plasma levels $(\mathrm{ng} / \mathrm{mL})$. (F-H)

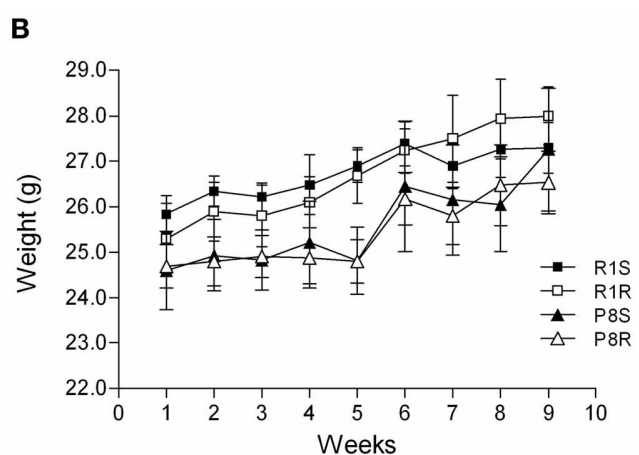

D

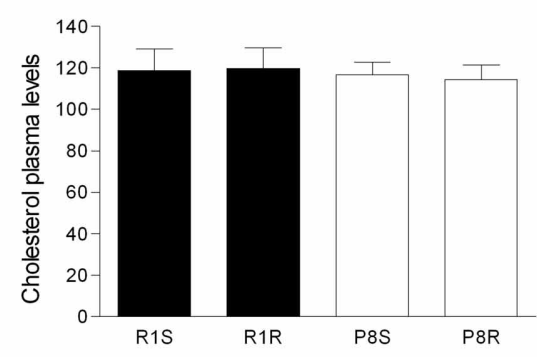

$\mathbf{F}$

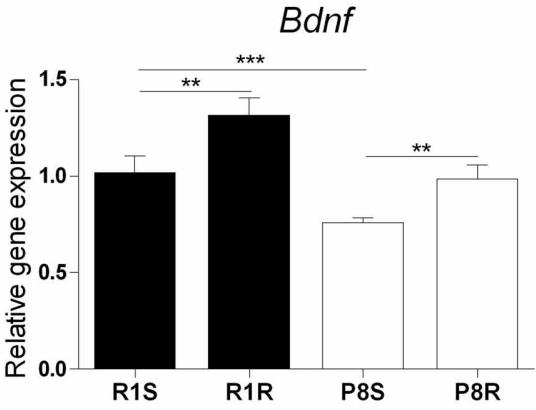

$\mathbf{H}$

Neuritin

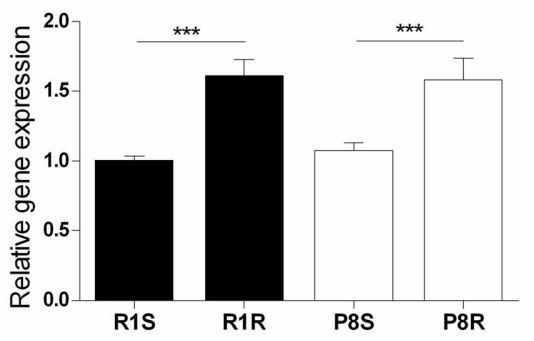

Gene expression of Bdnf (F), Trkb (G), and Neuritin (H). Gene expression was measured by real-time PCR analysis from hippocampal mRNA using TaqMan FAM-labeled specific probes and expressed relative to TBP ( $n=3-4$ /group for IGF1 and $n=5-8 /$ group for all other measures). Mean \pm standard error are represented; Two-Way ANOVA results are indicated as ${ }^{* *} p<0.01 ;{ }^{* * *} p<0.001$.
Voluntary exercise led to a significant decrease in Hdac3 gene expression exclusively in SAMP8 mice (Table 3, Figure 3E). ANOVA analysis showed a downregulation tendency for Hdac5 gene in exercised compared with sedentary SAMP8 mice, and this effect was found to be statistically significant by two-tailed Student's $t$-test for independent samples [P8R vs. P8S, Hdac3: $t_{(9)}=1.931, p=0.084$; Hdac5: $t_{(12)}=2.27, p=0.042$ ] (Table 3, Figure 3F). We did not detect any influence of the exercise 
Table 1 | Two-Way ANOVA analysis was used to compare plasma cholesterol, triglycerides and IGF1 levels and hippocampal expression of neurotrophic genes in sedentary and exercised 8-month-old SAMR1 and SAMP8 mice.

\begin{tabular}{|c|c|c|c|c|c|c|c|c|c|}
\hline & \multicolumn{9}{|c|}{ Two-way ANOVA analysis } \\
\hline & df. & $\boldsymbol{F}$ & p-value & df. & $\boldsymbol{F}$ & p-value & df. & $\boldsymbol{F}$ & $p$-value \\
\hline \multicolumn{10}{|l|}{ PLASMA } \\
\hline IGF1 & 1,10 & 19.716 & $<0.001$ & 1,10 & 0.008 & 0.932 & 1,10 & 0.528 & 0.484 \\
\hline \multicolumn{10}{|c|}{ HIPPOCAMPAL GENES } \\
\hline Bdnf & 1,22 & 12.16 & 0.002 & 1,22 & 15.285 & $<0.001$ & 1,22 & 0.233 & 0.634 \\
\hline $\operatorname{TrkB}$ & 1,21 & 8.239 & 0.009 & 1,21 & 3.729 & 0.067 & 1,21 & 2.26 & 0.148 \\
\hline Neuritin & 1,23 & 31.931 & $<0.001$ & 1,23 & 0.048 & 0.828 & 1,23 & 0.83 & 0.372 \\
\hline
\end{tabular}

Natural log, square root, and inverse transformation were applied to normalize plasma cholesterol, plasma triglycerides, and Neuritin distribution, respectively. $P$-values $<0.05$ were considered statistically significant. df stands for degrees of freedom. Bold values correspond to statistically significant $p$-values.

Table 2 | miRNAs significantly altered in SAMP8 mice and/or modulated by exercise.

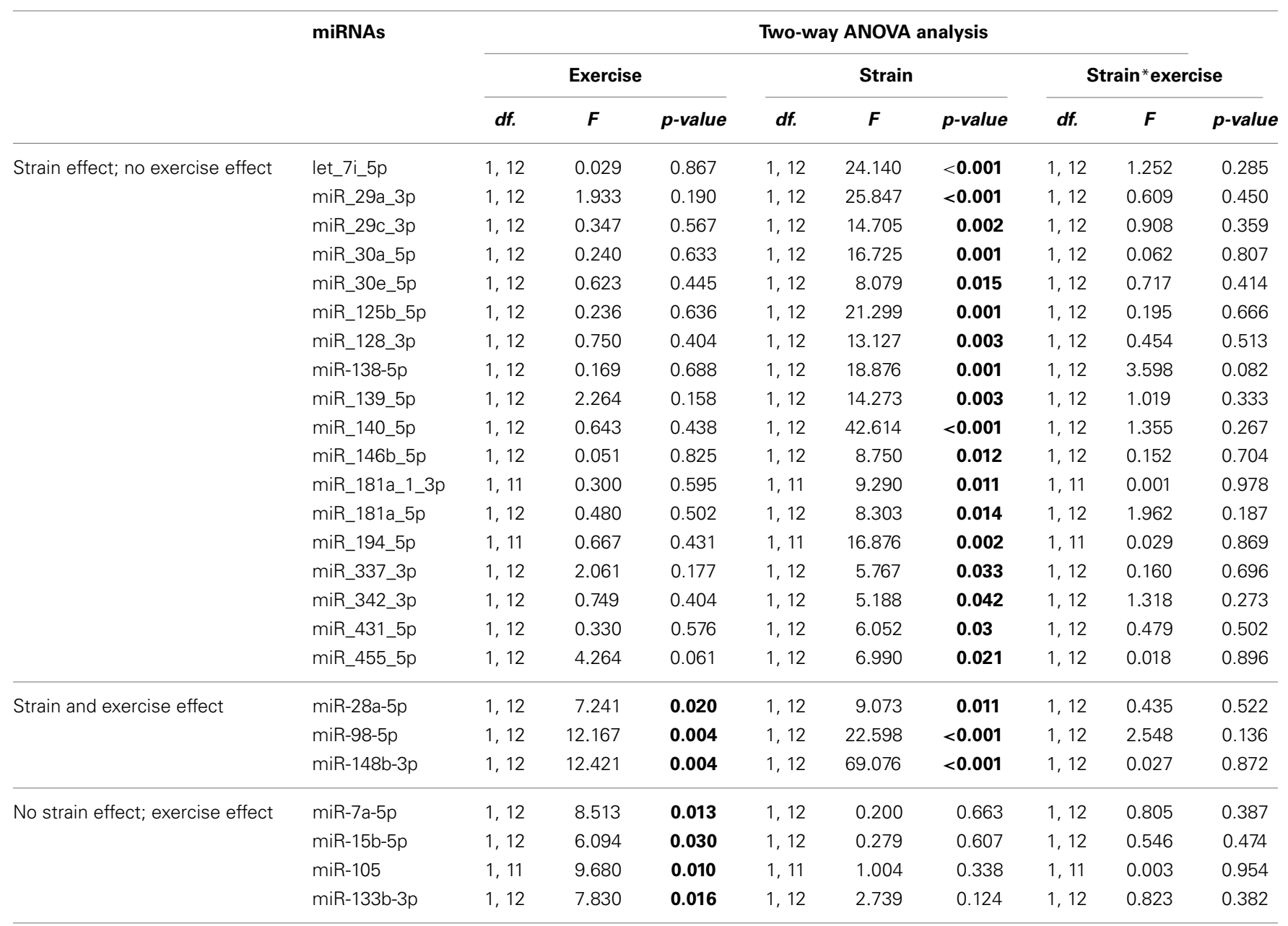

Two-Way ANOVA analysis was used to compare the hippocampal miRNAs gene expression in sedentary and exercised 8-month-old SAMR1 and SAMP8 mice. $P$-values $<0.05$ were considered statistically significant. df stands for degrees of freedom. Bold values correspond to statistically significant $p$-values. 


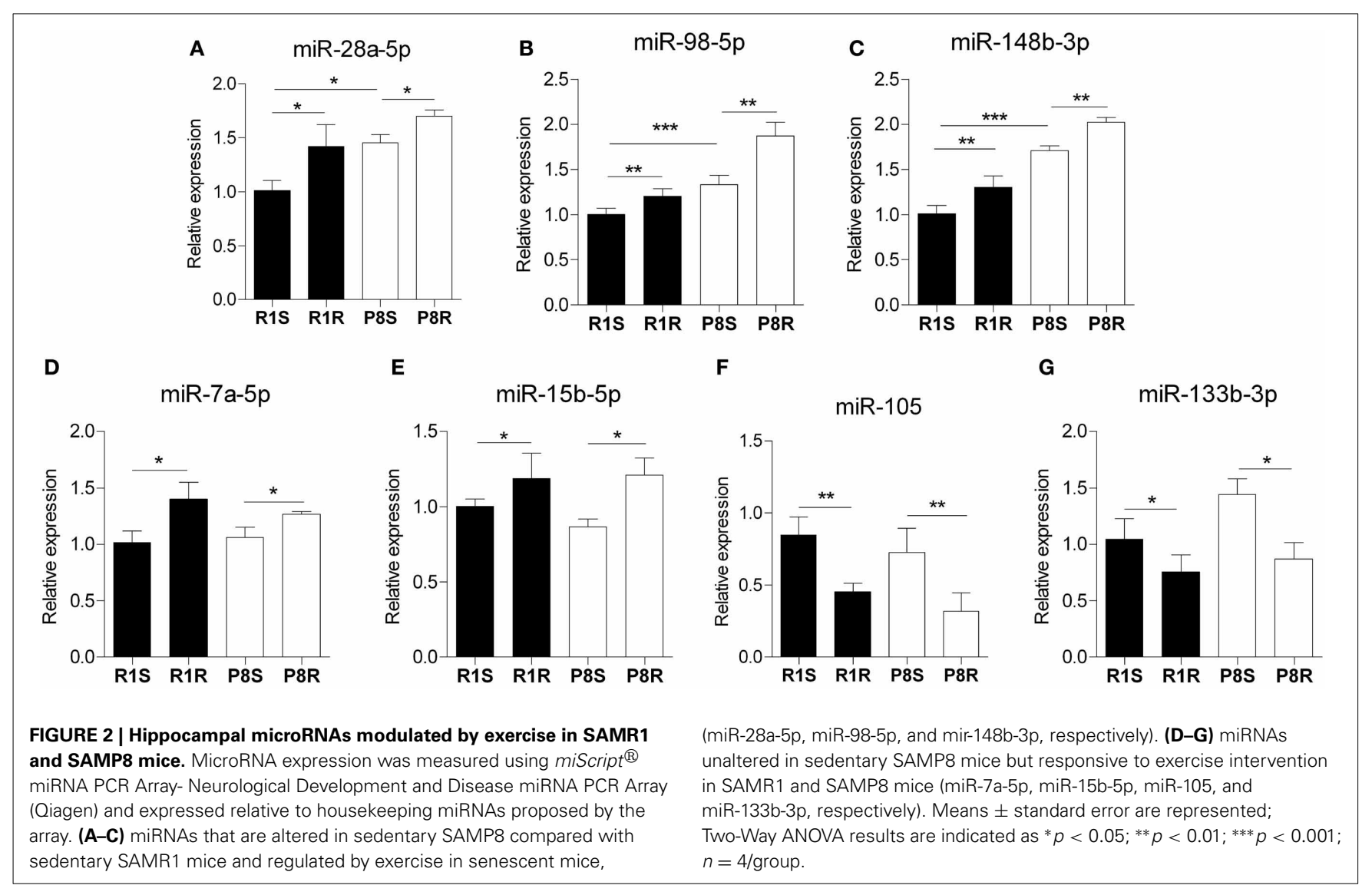

Table 3 | Two-Way ANOVA was used to compare the hippocampal expression of histone acetylation regulatory genes in sedentary and exercised 8-month-old SAMR1 and SAMP8 mice.

\begin{tabular}{|c|c|c|c|c|c|c|c|c|c|}
\hline \multirow[t]{2}{*}{ Hippocampal genes } & \multicolumn{9}{|c|}{ Two-way ANOVA analysis } \\
\hline & df. & $\boldsymbol{F}$ & $p$-value & $d f$. & $\boldsymbol{F}$ & $p$-value & $d f$. & $\boldsymbol{F}$ & p-value \\
\hline P300 & 1,23 & 0.106 & 0.748 & 1,23 & 1.261 & 0.273 & 1,23 & 2.942 & 0.1 \\
\hline Sirt1 & 1,23 & 0.091 & 0.765 & 1,23 & 8.369 & 0.008 & 1,23 & 0.041 & 0.841 \\
\hline Hdac3 & 1,23 & 0.054 & 0.818 & 1,23 & 0.383 & 0.542 & 1,23 & 5.249 & 0.031 \\
\hline Hdac5 & 1,23 & 1.414 & 0.246 & 1,23 & 14.639 & $<0.001$ & 1,23 & 3.104 & 0.091 \\
\hline Hdac6 & 1,23 & 1.482 & 0.236 & 1,23 & 21.536 & $<0.001$ & 1,23 & 0.453 & 0.508 \\
\hline
\end{tabular}

Quadratic transformation was applied to normalize Hdac3 distribution. P-values $<0.05$ were considered statistically significant. df stands for degrees of freedom. Bold values correspond to statistically significant p-values.

intervention on Sirt1, Hdac1, Hdac2, and Hdac6 gene expression (Table 3, Figures 3B-D,G).

Finally, we found that the global acetylation levels of histone $\mathrm{H} 3(\mathrm{H} 3 \mathrm{ac})$ were lower in sedentary SAMP8 than in SAMR1 mice [P8S vs. R1S, $t_{(6)}=3.929, p=0.008$ ] and significantly increased upon exercise only in the senescent mice [P8R vs. P8S, $\left.t_{(6)}=-3.399, p=0.019\right]$ (Figure 3H). In contrast, the acetylation of histone $4(\mathrm{H} 4 \mathrm{ac})$ did not show significant differences between groups (Figure 3I).

\section{DISCUSSION}

Here we explored the epigenetic alterations in the hippocampus of SAMP8 female mouse and the modulatory effect of voluntary physical exercise on the expression of several miRNAS, histone deacetylase genes and in the global acetylation level of histone $\mathrm{H} 3$.

Our data and those of others (Liang et al., 2009) suggest that miRNAs are involved in the down-regulation of target genes that control accelerated senescence. Indeed, we found a general upregulation pattern of miRNAs in the hippocampus SAMP8 

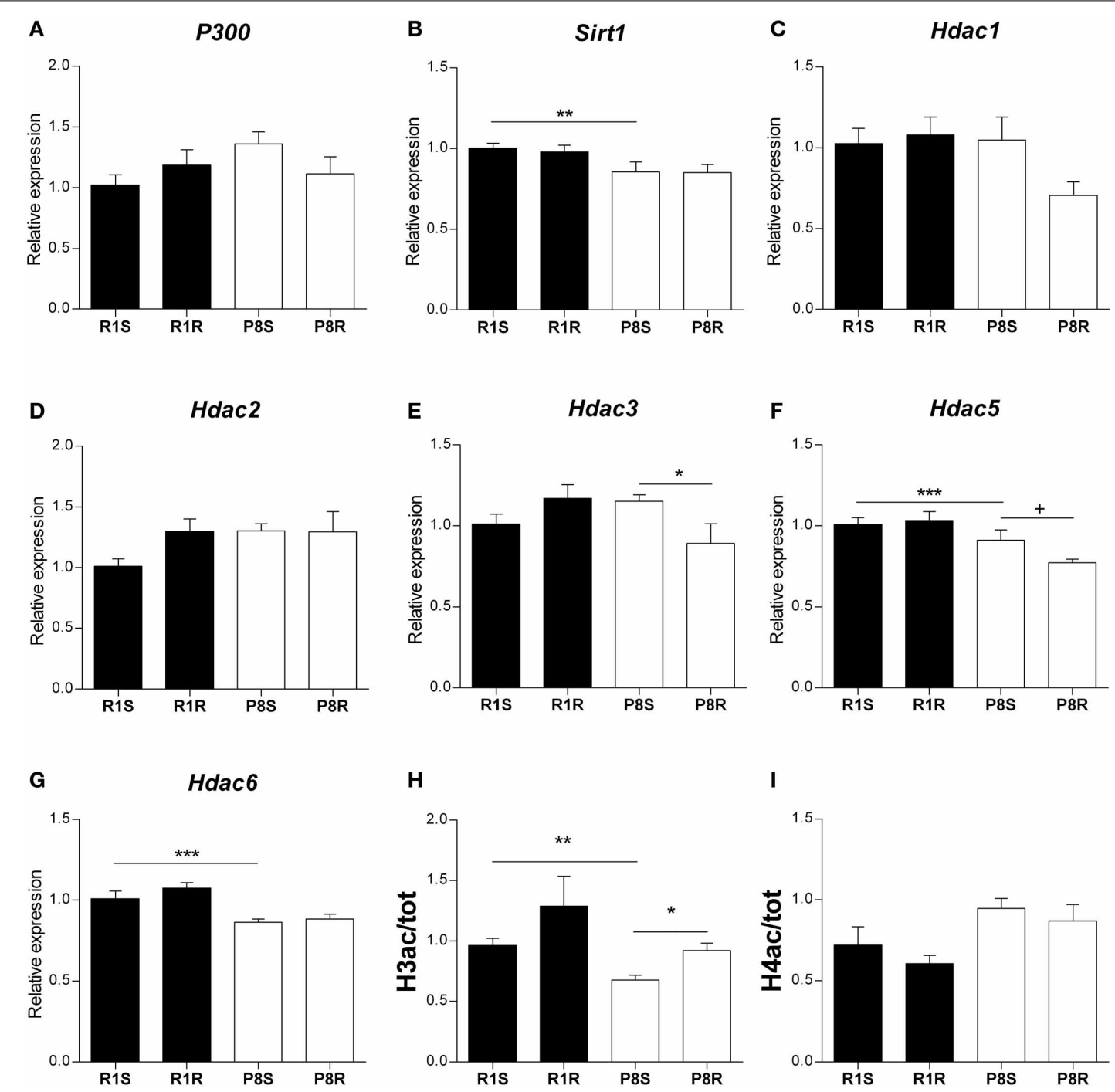

FIGURE 3 | Histone acetylation regulatory genes and global histone modifications in hippocampus of sedentary and exercised 8 months-old SAMR1 and SAMP8 mice. Gene expression was measured by real-time PCR analysis from hippocampal mRNA using TaqMan FAM-labeled specific probes and expressed relative to TBP ( $n=5-8 /$ group). (A) Histone acetyltransferase P-300. (B) Sirtuin 1. (C-G) Histone deacetylase 1-6, respectively. Means \pm standard error are represented; Two-Way ANOVA results are indicated as
${ }^{*} p<0.05 ;{ }^{* *} p<0.01 ;{ }^{* *} p<0.001$. $(\mathbf{H}, \mathbf{I})$ Global acetylation levels of histone H3 (H) and histone H4 (I) in hippocampus from sedentary and exercised SAMR1 and SAMP8 mice. Specific bands from Western blot were quantified by scanning densitometry ( $n=4 /$ group). Histone modifications were corrected by total histone and results were analyzed by two-tailed Student's $t$-test for independent samples. Means \pm standard error are represented $\left({ }^{+} p<0.1 ;{ }^{*} p<0.05 ;{ }^{* *} p<0.01 ;{ }^{* *} p<0.001\right)$. compared with SAMR1 mice. Most of these miRNAs have also been found dysregulated in different tissues from AD patients. Notably, our study highlights the upregulation of miR-30e-5p, miR-125b-5p, and miR-128-3p as common epigenetic features in the hippocampus of SAMP8 mice and post-mortem hippocampus from AD patients. Moreover, our results support bioinformatic data by Cheng et al. who have recently predicted from a whole genome microarray study that miR-125b-5p may be involved in the brain aging phenotype of SAMP8 mice (Cheng et al., 2013a). Therefore, these miRNAs emerge as potential AD biomarkers and our data provide further support for the suitability of the SAMP8 model for future studies to explore their role on the onset and progression of AD. Supplementary Table 3 (S3) summarizes the available literature regarding the brain distribution and function of the miRNAs that we found altered in SAMP8 compared with SAMR1 sedentary mice. Bioinformatic pathway analysis (DIANA-miRPath v.2.1) indicates that these miRNAs are involved in neural processes such as neurotransmitters synapses (acetylcholine, glutamate, dopamine), long-term potentiation, axon guidance and neurotrophin signaling (S5).

Exercise training led to the increase of IGF1 in plasma and the upregulation of BDNF and other neurogenic factors in hippocampus of SAMP8 and SAMR1 strains (Figure 1). These data confirm that the intervention used in our study was effective 
as such effects have previously been reported in a variety of rodent models in response to exercise (Saltiel and Kahn, 2001; Llorens-Martin et al., 2010; Chang et al., 2011; Kaliman et al., 2011; Sakurai et al., 2011; Higashi et al., 2012; Alvarez-Lopez et al., 2013). We found that miR-28a-5p, miR-98a-5p, miR-148b$3 p$ were altered in sedentary SAMP8 compared with SAMR1 mice and changed their expression levels in response to exercise (putative aging markers responsive to exercise). On the other hand, miR-7a-5p, miR-15b-5p, miR-105, miR-133b-3p, which were similarly expressed in SAMP8 and SAMR1 mice, were modulated by exercise in both strains (putative markers of exercise unrelated to aging). The data available on the function and expression of these miRNAs in the CNS are summarized in Supplementary Table 4 (S4). Further study is warranted to explore the precise mechanistic links between these miRNAs and the protective central effects of physical exercise. In this context, a prediction through bioinformatic pathway analysis for multiple miRNA effect indicates that these exercise-responsive miRNAs are involved in the regulation of PI-3-kinase-Akt, focal adhesion, insulin, mTOR and MapK signaling pathways, all of which are modulated in the brain by exercise (Shen et al., 2001; Tong et al., 2001; Bruel-Jungerman et al., 2009; Muller et al., 2011; Elfving et al., 2013) (S6).

Both the sedentary and exercised SAMP8 mice showed altered expression patterns of protein deacetylases with reported functions in the aging brain and AD such as Hdac6, Sirt1, Hdac3, and Hdac5. We found a downregulation of histone deacetylase Hdac6 in the hippocampus of sedentary SAMP8 mice. HDAC6 specific inhibitors have been described as potential therapeutic approaches to rescue the neurodegeneration, however an induction of HDAC6 was reported to facilitate the autophagy of misfolded proteins and aggregates of A 342 and p-tau (Simoes-Pires et al., 2013). Further research is required to better understand the still controversial role of HDAC6 and our data indicate that the SAMP8 mice may represent a suitable model for this purpose. We also found a downregulation of the protein deacetylase Sirt1 in the hippocampus of SAMP8 mice, supporting the notion that decreased Sirt1 expression is a feature of the accelerated brain aging and neurodegeneration (Pallas et al., 2008b; Duan, 2013). However, we did not find a modulation of Sirt1 mRNA levels in response to the running intervention in contrast to previous findings using other experimental models (Ferrara et al., 2008; Dumke et al., 2009; Koltai et al., 2010).

Our data suggest that exercise may exert some of its reported beneficial effects on SAMP8 cognitive performance through Hdac3 downregulation, a mechanism involved in long-term memory enhancement (Mcquown et al., 2011) and in the reversion of contextual memory deficits in a mouse model of $\mathrm{AD}$ (Fischer et al., 2007; Kilgore et al., 2010).

It has previously been reported that exercise activates $B d n f$ transcription through Hdac5 downregulation (Gomez-Pinilla et al., 2011). Therefore, the downregulation of Hdac5 expression in the exercised SAMP8 mice may be at least in part responsible for the observed $B d n f$ upregulation (Figure 2). Finally, global acetylation levels of histone $\mathrm{H} 3$ ( $\mathrm{H} 3 \mathrm{ac}$ ) were increased after the exercise intervention in the SAMP8 mice suggesting that HDAC gene downregulation had some impact on chromatin remodeling.
Notably, partial correlation analyses revealed a negative association between the modification $\mathrm{H} 3 \mathrm{ac}$ and $\mathrm{Hdac} 3$ gene expression $\left[\rho_{(11)}=-0.721, p<0.01\right]$.

As a whole, our study highlights some common epigenetic features in hippocampus of SAMP8 mice and human AD, and provides further support for the suitability of this experimental model for future epigenetic studies regarding the onset and progression of $\mathrm{AD}$. Among them, miRNAs emerge as potentially valuable biomarkers for the development of new therapeutic strategies for senescence and neurodegeneration. Moreover, our data suggest a positive impact of voluntary exercise in reversing some epigenetic and transcriptional alterations associated with the aging brain, and reinforces the prevailing concept that physical training is a promising therapeutic strategy for neurodegenerative diseases such as $\mathrm{AD}$.

\section{ACKNOWLEDGMENTS}

This study was supported by grant SAF2010-15050 (Perla Kaliman) from the Spanish Ministry of Economy and Competitiveness (MINECO) with the collaboration of grants DPS2008-06998-C02 (Merce Pallàs and Rosa M. Escorihuela); CSD2010-45 (Coral Sanfeliu) and SAF2012-39852 (Merce Pallàs and Coral Sanfeliu) from MINECO. Marta Cosín-Tomás is supported by a predoctoral fellowship from MINECO (FPU 2013); Jaume F. Lalanza and María J. Alvarez-López were supported by a predoctoral fellowship from the Generalitat de Catalunya (FI-DGR 2011).

\section{SUPPLEMENTARY MATERIAL}

The Supplementary Material for this article can be found online at: http://www.frontiersin.org/journal/10.3389/fnagi.2014. 00051/abstract

\section{REFERENCES}

Abel, J. L., and Rissman, E. F. (2013). Running-induced epigenetic and gene expression changes in the adolescent brain. Int. J. Dev. Neurosci. 31, 382-390. doi: 10.1016/j.ijdevneu.2012.11.002

Akbarian, S., Beeri, M. S., and Haroutunian, V. (2013). Epigenetic determinants of healthy and diseased brain aging and cognition. JAMA Neurol. 70, 711-718. doi: 10.1001/jamaneurol.2013.1459

Alvarez-Lopez, M. J., Castro-Freire, M., Cosin-Tomas, M., Sanchez-Roige, S., Lalanza, J. F., Del Valle, J., et al. (2013). Long-term exercise modulates hippocampal gene expression in senescent female mice. J. Alzheimers Dis. 33, 1177-1190. doi: 10.3233/JAD-121264

Arendt, T. (2009). Synaptic degeneration in Alzheimer's disease. Acta Neuropathol. 118, 167-179. doi: 10.1007/s00401-009-0536-x

Bartel, D. P. (2009). MicroRNAs: target recognition and regulatory functions. Cell 136, 215-233. doi: 10.1016/j.cell.2009.01.002

Bentwich, I., Avniel, A., Karov, Y., Aharonov, R., Gilad, S., Barad, O., et al. (2005). Identification of hundreds of conserved and nonconserved human microRNAs. Nat. Genet. 37, 766-770. doi: 10.1038/ng1590

Bruel-Jungerman, E., Veyrac, A., Dufour, F., Horwood, J., Laroche, S., and Davis, S. (2009). Inhibition of PI3K-Akt signaling blocks exercise-mediated enhancement of adult neurogenesis and synaptic plasticity in the dentate gyrus. PLoS ONE 4:e7901. doi: 10.1371/journal.pone.0007901

Chandramohan, Y., Droste, S. K., Arthur, J. S., and Reul, J. M. (2008). The forced swimming-induced behavioural immobility response involves histone $\mathrm{H} 3$ phospho-acetylation and c-Fos induction in dentate gyrus granule neurons via activation of the N-methyl-D-aspartate/extracellular signal-regulated kinase/mitogen- and stress-activated kinase signalling pathway. Eur. J. Neurosci. 27, 2701-2713. doi: 10.1111/j.1460-9568.2008.06230.x 
Chang, H. C., Yang, Y. R., Wang, P. S., Kuo, C. H., and Wang, R. Y. (2011). Insulin-like growth factor I signaling for brain recovery and exercise ability in brain ischemic rats. Med. Sci. Sports Exerc. 43, 2274-2280. doi: 10.1249/MSS.0b013e318223b5d9

Cheng, X. R., Cui, X. L., Zheng, Y., Zhang, G. R., Li, P., Huang, H., et al. (2013a). Nodes and biological processes identified on the basis of network analysis in the brain of the senescence accelerated mice as an Alzheimer's disease animal model. Front. Aging Neurosci. 5:65. doi: 10.3389/fnagi.2013.00065

Cheng, X. R., Zhou, W. X., and Zhang, Y. X. (2013b). The behavioral, pathological and therapeutic features of the senescence-accelerated mouse prone 8 strain as an Alzheimer's disease animal model. Ageing Res. Rev. 13C, 13-37. doi: 10.1016/j.arr.2013.10.002

Cogswell, J. P., Ward, J., Taylor, I. A., Waters, M., Shi, Y., Cannon, B., et al. (2008). Identification of miRNA changes in Alzheimer's disease brain and CSF yields putative biomarkers and insights into disease pathways. J. Alzheimers Dis. 14, 27-41.

Collins, A., Hill, L. E., Chandramohan, Y., Whitcomb, D., Droste, S. K., and Reul, J. M. (2009). Exercise improves cognitive responses to psychological stress through enhancement of epigenetic mechanisms and gene expression in the dentate gyrus. PLoS ONE 4:e4330. doi: 10.1371/journal.pone.0004330

Crepaldi, L., and Riccio, A. (2009). Chromatin learns to behave. Epigenetics 4, 23-26. doi: 10.4161/epi.4.1.7604

Dang, W., Steffen, K. K., Perry, R., Dorsey, J. A., Johnson, F. B., Shilatifard, A., et al. (2009). Histone H4 lysine 16 acetylation regulates cellular lifespan. Nature 459, 802-807. doi: 10.1038/nature08085

Del Valle, J., Duran-Vilaregut, J., Manich, G., Pallas, M., Camins, A., Vilaplana, J., et al. (2011). Cerebral amyloid angiopathy, blood-brain barrier disruption and amyloid accumulation in SAMP8 mice. Neurodegener. Dis. 8, 421-429. doi: 10.1159/000324757

Di Bernardo, G., Cipollaro, M., and Galderisi, U. (2012). Chromatin modification and senescence. Curr. Pharm. Des. 18, 1686-1693. doi: 10.2174/138161212799859693

Dishman, R. K., Berthoud, H. R., Booth, F. W., Cotman, C. W., Edgerton, V. R., Fleshner, M. R., et al. (2006). Neurobiology of exercise. Obesity (Silver Spring) 14, 345-356. doi: 10.1038/oby.2006.46

Duan, W. (2013). Sirtuins: from metabolic regulation to brain aging. Front. Aging Neurosci. 5:36. doi: 10.3389/fnagi.2013.00036

Dumke, C. L., Mark Davis, J., Angela Murphy, E., Nieman, D. C., Carmichael, M. D., Quindry, J. C., et al. (2009). Successive bouts of cycling stimulates genes associated with mitochondrial biogenesis. Eur. J. Appl. Physiol. 107, 419-427. doi: 10.1007/s00421-009-1143-1

Elfving, B., Christensen, T., Ratner, C., Wienecke, J., and Klein, A. B. (2013). Transient activation of mTOR following forced treadmill exercise in rats. Synapse 67, 620-625. doi: 10.1002/syn.21668

Ferrara, N., Rinaldi, B., Corbi, G., Conti, V., Stiuso, P., Boccuti, S., et al. (2008). Exercise training promotes SIRT1 activity in aged rats. Rejuvenation Res. 11, 139-150. doi: 10.1089/rej.2007.0576

Fischer, A., Sananbenesi, F., Wang, X., Dobbin, M., and Tsai, L. H. (2007). Recovery of learning and memory is associated with chromatin remodelling. Nature 447, 178-182. doi: 10.1038/nature05772

Goldie, B. J., and Cairns, M. J. (2012). Post-transcriptional trafficking and regulation of neuronal gene expression. Mol. Neurobiol. 45, 99-108. doi: 10.1007/s12035-011-8222-0

Gomez-Pinilla, F., Vaynman, S., and Ying, Z. (2008). Brain-derived neurotrophic factor functions as a metabotrophin to mediate the effects of exercise on cognition. Eur. J. Neurosci. 28, 2278-2287. doi: 10.1111/j.1460-9568.2008.06524.x

Gomez-Pinilla, F., Zhuang, Y., Feng, J., Ying, Z., and Fan, G. (2011). Exercise impacts brain-derived neurotrophic factor plasticity by engaging mechanisms of epigenetic regulation. Eur. J. Neurosci. 33, 383-390. doi: 10.1111/j.14609568.2010.07508.x

Greer, E. L., Maures, T. J., Hauswirth, A. G., Green, E. M., Leeman, D. S., Maro, G. S., et al. (2010). Members of the H3K4 trimethylation complex regulate lifespan in a germline-dependent manner in C. elegans. Nature 466, 383-387. doi: 10.1038 /nature09195

Hebert, S. S., Horre, K., Nicolai, L., Papadopoulou, A. S., Mandemakers, W., Silahtaroglu, A. N., et al. (2008). Loss of microRNA cluster miR-29a/b1 in sporadic Alzheimer's disease correlates with increased BACE1/betasecretase expression. Proc. Natl. Acad. Sci. U.S.A. 105, 6415-6420. doi: 10.1073/pnas.0710263105
Higashi, Y., Sukhanov, S., Anwar, A., Shai, S. Y., and Delafontaine, P. (2012). Aging, atherosclerosis, and IGF-1. J. Gerontol. A Biol. Sci. Med. Sci. 67, 626-639. doi: $10.1093 /$ gerona/gls102

Huidobro, C., Fernandez, A. F., and Fraga, M. F. (2013). Aging epigenetics: causes and consequences. Mol. Aspects Med. 34, 765-781. doi: 10.1016/j.mam.2012.06.006

Jaenisch, R., and Bird, A. (2003). Epigenetic regulation of gene expression: how the genome integrates intrinsic and environmental signals. Nat. Genet. 33(Suppl.), 245-254. doi: 10.1038/ng1089

Jakovcevski, M., and Akbarian, S. (2012). Epigenetic mechanisms in neurological disease. Nat. Med. 18, 1194-1204. doi: 10.1038/nm.2828

Jung, H. J., and Suh, Y. (2012). microRNA in aging: from discovery to biology. Curr. Genomics 13, 548-557. doi: 10.2174/138920212803251436

Kaliman, P., Parrizas, M., Lalanza, J. F., Camins, A., Escorihuela, R. M., and Pallas, M. (2011). Neurophysiological and epigenetic effects of physical exercise on the aging process. Ageing Res. Rev. 10, 475-486. doi: 10.1016/j.arr.2011.05.002

Kilgore, M., Miller, C. A., Fass, D. M., Hennig, K. M., Haggarty, S. J., Sweatt, J. D., et al. (2010). Inhibitors of class 1 histone deacetylases reverse contextual memory deficits in a mouse model of Alzheimer's disease. Neuropsychopharmacology 35, 870-880. doi: 10.1038/npp.2009.197

Koltai, E., Szabo, Z., Atalay, M., Boldogh, I., Naito, H., Goto, S., et al. (2010). Exercise alters SIRT1, SIRT6, NAD and NAMPT levels in skeletal muscle of aged rats. Mech. Ageing Dev. 131, 21-28. doi: 10.1016/j.mad.2009.11.002

Lau, P., Bossers, K., Janky, R., Salta, E., Frigerio, C. S., Barbash, S., et al. (2013). Alteration of the microRNA network during the progression of Alzheimer's disease. EMBO Mol. Med. 5, 1613-1634. doi: 10.1002/emmm.201201974

Legube, G., and Trouche, D. (2003). Regulating histone acetyltransferases and deacetylases. EMBO Rep. 4, 944-947. doi: 10.1038/sj.embor.embor941

Liang, R., Bates, D. J., and Wang, E. (2009). Epigenetic control of microRNA expression and aging. Curr. Genomics 10, 184-193. doi: 10.2174/138920209788185225

Llorens-Martin, M. V., Rueda, N., Tejeda, G. S., Florez, J., Trejo, J. L., and MartinezCue, C. (2010). Effects of voluntary physical exercise on adult hippocampal neurogenesis and behavior of Ts65Dn mice, a model of Down syndrome. Neuroscience 171, 1228-1240. doi: 10.1016/j.neuroscience.2010.09.043

Lopez-Otin, C., Blasco, M. A., Partridge, L., Serrano, M., and Kroemer, G. (2013). The hallmarks of aging. Cell 153, 1194-1217. doi: 10.1016/j.cell.2013.05.039

Lovatel, G. A., Elsner, V. R., Bertoldi, K., Vanzella, C., Moyses Fdos, S., Vizuete, A., et al. (2013). Treadmill exercise induces age-related changes in aversive memory, neuroinflammatory and epigenetic processes in the rat hippocampus. Neurobiol. Learn. Mem. 101, 94-102. doi: 10.1016/j.nlm.2013.01.007

Lukiw, W. J. (2007). Micro-RNA speciation in fetal, adult and Alzheimer's disease hippocampus. Neuroreport 18, 297-300. doi: 10.1097/WNR.0b013e3280148e8b

Maes, O. C., Chertkow, H. M., Wang, E., and Schipper, H. M. (2009). microRNA: implications for Alzheimer disease and other human CNS disorders. Curr. Genomics 10, 154-168. doi: 10.2174/138920209788185252

Mastroeni, D., Grover, A., Delvaux, E., Whiteside, C., Coleman, P. D., and Rogers, J. (2011). Epigenetic mechanisms in Alzheimer's disease. Neurobiol. Aging 32, 1161-1180. doi: 10.1016/j.neurobiolaging.2010.08.017

Mcquown, S. C., Barrett, R. M., Matheos, D. P., Post, R. J., Rogge, G. A., Alenghat, T., et al. (2011). HDAC3 is a critical negative regulator of long-term memory formation. J. Neurosci. 31, 764-774. doi: 10.1523/JNEUROSCI.5052-10.2011

Min, S. W., Cho, S. H., Zhou, Y., Schroeder, S., Haroutunian, V., Seeley, W. W., et al. (2010). Acetylation of tau inhibits its degradation and contributes to tauopathy. Neuron 67, 953-966. doi: 10.1016/j.neuron.2010.08.044

Morley, J. E., Armbrecht, H. J., Farr, S. A., and Kumar, V. B. (2012a). The senescence accelerated mouse (SAMP8) as a model for oxidative stress and Alzheimer's disease. Biochim. Biophys. Acta 1822, 650-656. doi: 10.1016/j.bbadis.2011.11.015

Morley, J. E., Farr, S. A., Kumar, V. B., and Armbrecht, H. J. (2012b). The SAMP8 mouse: a model to develop therapeutic interventions for Alzheimer's disease. Curr. Pharm. Des. 18, 1123-1130. doi: 10.2174/138161212799315795

Muller, A. P., Gnoatto, J., Moreira, J. D., Zimmer, E. R., Haas, C. B., Lulhier, F., et al. (2011). Exercise increases insulin signaling in the hippocampus: physiological effects and pharmacological impact of intracerebroventricular insulin administration in mice. Hippocampus 21, 1082-1092. doi: 10.1002/hipo.20822

Pallas, M., Camins, A., Smith, M. A., Perry, G., Lee, H. G., and Casadesus, G. (2008a). From aging to Alzheimer's disease: unveiling "the switch" with the senescence-accelerated mouse model (SAMP8). J. Alzheimers Dis. 15, 615-624.

Pallas, M., Pizarro, J. G., Gutierrez-Cuesta, J., Crespo-Biel, N., Alvira, D., Tajes, M., et al. (2008b). Modulation of SIRT1 expression in different 
neurodegenerative models and human pathologies. Neuroscience 154, 1388-1397. doi: 10.1016/j.neuroscience.2008.04.065

Saha, R. N., and Pahan, K. (2006). HATs and HDACs in neurodegeneration: a tale of disconcerted acetylation homeostasis. Cell Death Differ. 13, 539-550. doi: 10.1038/sj.cdd.4401769

Sakurai, T., Ogasawara, J., Kizaki, T., Ishibashi, Y., Fujiwara, T., Akagawa, K., et al. (2011). Involvement of leucine zipper transcription factor-like protein 1 (Lztfl1) in the attenuation of cognitive impairment by exercise training. Biochem. Biophys. Res. Commun. 416, 125-129. doi: 10.1016/j.bbrc.2011.11.008

Saltiel, A. R., and Kahn, C. R. (2001). Insulin signalling and the regulation of glucose and lipid metabolism. Nature 414, 799-806. doi: 10.1038/414799a

Scheff, S. W., Price, D. A., Schmitt, F. A., Dekosky, S. T., and Mufson, E. J. (2007). Synaptic alterations in CA1 in mild Alzheimer disease and mild cognitive impairment. Neurology 68, 1501-1508. doi: 10.1212/01.wnl.0000260698.46517.8f

Shen, H., Tong, L., Balazs, R., and Cotman, C. W. (2001). Physical activity elicits sustained activation of the cyclic AMP response element-binding protein and mitogen-activated protein kinase in the rat hippocampus. Neuroscience 107, 219-229. doi: 10.1016/S0306-4522(01)00315-3

Siebold, A. P., Banerjee, R., Tie, F., Kiss, D. L., Moskowitz, J., and Harte, P. J. (2010). Polycomb repressive complex 2 and trithorax modulate drosophila longevity and stress resistance. Proc. Natl. Acad. Sci. U.S.A. 107, 169-174. doi: 10.1073/pnas.0907739107

Simoes-Pires, C., Zwick, V., Nurisso, A., Schenker, E., Carrupt, P. A., and Cuendet, M. (2013). HDAC6 as a target for neurodegenerative diseases: what makes it different from the other HDACs? Mol. Neurodegener. 8:7. doi: 10.1186/17501326-8-7

Takeda, T. (2009). Senescence-accelerated mouse (SAM) with special references to neurodegeneration models, SAMP8 and SAMP10 mice. Neurochem. Res. 34, 639-659. doi: 10.1007/s11064-009-9922-y

Takeda, T., Hosokawa, M., and Higuchi, K. (1991). Senescence-accelerated mouse (SAM): a novel murine model of accelerated senescence. J. Am. Geriatr. Soc. 39, 911-919.

Tammen, S. A., Friso, S., and Choi, S. W. (2013). Epigenetics: the link between nature and nurture. Mol. Aspects Med. 34, 753-764. doi: 10.1016/j.mam.2012.07.018

Tong, L., Shen, H., Perreau, V. M., Balazs, R., and Cotman, C. W. (2001). Effects of exercise on gene-expression profile in the rat hippocampus. Neurobiol. Dis. 8, 1046-1056. doi: 10.1006/nbdi.2001.0427
Trejo, J. L., Llorens-Martin, M. V., and Torres-Aleman, I. (2008). The effects of exercise on spatial learning and anxiety-like behavior are mediated by an IGF-I-dependent mechanism related to hippocampal neurogenesis. Mol. Cell. Neurosci. 37, 402-411. doi: 10.1016/j.mcn.2007.10.016

Van Den Hove, D. L., Kompotis, K., Lardenoije, R., Kenis, G., Mill, J., Steinbusch, H. W., et al. (2014). Epigenetically regulated microRNAs in Alzheimer's disease. Neurobiol. Aging 35, 731-745. doi: 10.1016/j.neurobiolaging.2013.10.082

Wang, H., Lian, K., Han, B., Wang, Y., Kuo, S. H., Geng, Y., et al. (2013). Age-related alterations in the metabolic profile in the hippocampus of the senescenceaccelerated mouse prone 8: a spontaneous Alzheimer's disease mouse model. J. Alzheimers Dis. 39, 841-848. doi: 10.3233/JAD-131463

Wang, W., Kwon, E. J., and Tsai, L. H. (2012). MicroRNAs in learning, memory, and neurological diseases. Learn. Mem. 19, 359-368. doi: 10.1101/lm.026492.112

Wang, W. X., Huang, Q., Hu, Y., Stromberg, A. J., and Nelson, P. T. (2011). Patterns of microRNA expression in normal and early Alzheimer's disease human temporal cortex: white matter versus gray matter. Acta Neuropathol. 121, 193-205. doi: 10.1007/s00401-010-0756-0

Yang, Z., Vilkaitis, G., Yu, B., Klimasauskas, S., and Chen, X., (2007). Approaches for studying microRNA and small interfering RNA methylation in vitro and in vivo. Methods Enzymol. 427, 139-154. doi: 10.1016/S0076-6879(07)27008-9

Conflict of Interest Statement: The authors declare that the research was conducted in the absence of any commercial or financial relationships that could be construed as a potential conflict of interest.

Received: 20 January 2014; paper pending published: 09 February 2014; accepted: 03 March 2014; published online: 20 March 2014.

Citation: Cosín-Tomás M, Alvarez-López MJ, Sanchez-Roige S, Lalanza JF, Bayod S, Sanfeliu C, Pallàs M, Escorihuela RM and Kaliman P (2014) Epigenetic alterations in hippocampus of SAMP8 senescent mice and modulation by voluntary physical exercise. Front. Aging Neurosci. 6:51. doi: 10.3389/fnagi.2014.00051

This article was submitted to the journal Frontiers in Aging Neuroscience.

Copyright (c) 2014 Cosín-Tomás, Alvarez-López, Sanchez-Roige, Lalanza, Bayod, Sanfeliu, Pallàs, Escorihuela and Kaliman. This is an open-access article distributed under the terms of the Creative Commons Attribution License (CC BY). The use, distribution or reproduction in other forums is permitted, provided the original author(s) or licensor are credited and that the original publication in this journal is cited, in accordance with accepted academic practice. No use, distribution or reproduction is permitted which does not comply with these terms. 